\title{
Grazie a una grande squadra
}

\author{
Annamaria Colao ${ }^{1} \cdot$ Andrea Lenzi $^{2} \cdot$ Francesco Trimarchi $^{3}$
}

๑) Springer International Publishing AG, part of Springer Nature 2018

La Direzione Scientifica de l'Endocrinologo ringrazia sentitamente, per il determinante contributo all'ulteriore crescita della Rivista che si è ormai affermata (non solo nella Comunità endocrinologica) come indispensabile strumento di diffusione culturale e di aggiornamento professionale, i Colleghi che si sono avvicendati come Caporedattori, Coordinatori o Responsabili di Sezione del Comitato scientifico di Redazione o di Rubriche:

Maria Luisa Appetecchia

Emanuela Arvat

Giancarlo Balercia

Riccardo Bonadonna

Luisella Cianferotti

Rossella Elisei

Carla Giordano

Antonio La Marca

Nicola Palestini

Francesca Pecori Giraldi

Alfredo Pontecorvi

Antonio Sinisi

Anna Spada

Maria Laura Tanda

F. Trimarchi trimarki@unime.it

1 Napoli, Italia

2 Roma, Italia

3 Messina, Italia 